\title{
Adsorção de Poli(acrilamida- $b-\mathrm{N}, \mathrm{N}$-dihexilacrilamida) sobre Arenito: Determinação das Condições para se Atingir o Equilíbrio Real
}

\author{
Ana Maria S. Maia, Rosangela B. Garcia \\ Departamento de Química, UFRN
}

\begin{abstract}
Resumo: Durante as últimas décadas, o interesse em polímeros anfifílicos tem aumentado continuamente. Neste trabalho, o comportamento do copolímero hidrofílico/hidrofóbico poli(acrilamida- $b$-N,N-dihexilacrilamida) (PADHA) na interface sólido/líquido foi estudado para se quantificar a adsorção quando o equilíbrio do sistema é atingido. Na interface água/arenito, a isoterma de adsorção para a PADHA mostrou uma forma incomum, caracterizada pela ausência de uma região de patamar e um aumento contínuo na quantidade adsorvida com a concentração polimérica. Esta forma de adsorção particular poderia ser explicada pela formação de múltiplas camadas devido a associações hidrofóbicas, através das quais as camadas adsorvidas seriam formadas, em parte, por algumas cadeias que não estão diretamente em contato com a superfície sólida.

Palavras-chave: Adsorção, copolímero anfifilico, poliacrilamida.

\section{Poly(acrylamide-b-N,N-dihexylacrylamide) Adsorption on Sandstone: Determination of the Conditions to Reach the Real Equilibrium}

Abstract: During the last few decades, interest in amphiphilic polymers has increased steadily. In this work, the behavior of the hydrophilic/hydrophobic copolymer poly(acrylamide- $b$-N,N-dihexylacrylamide) (PADHA) at a solid/liquid interface has been studied to quantify adsorption at equilibrium. At the water/sandstone interface, the adsorption isotherm for PADHA show an unusual profile, characterized by the absence of a "plateau" region and a monotonic increase in the adsorbed amount with polymer concentration in the bulk. This particular adsorption profile could be explained by the formation of multiple layers caused by hydrophobic associations, the adsorbed layers being partly formed by chains that are not directly in contact with the surface.

Keywords: Adsorption, amphiphilic copolymer, polyacrylamide.

\section{Introdução}

Polímeros hidrofobicamente modificados e que são solúveis em água têm encontrado um grande número de aplicações práticas nos últimos $20 \operatorname{anos}^{[1]}$. Devido à alta capacidade que possuem de aumentar a viscosidade do meio aquoso, eles podem ser utilizados nas mais diversas áreas, como, por exemplo, em tintas, cosméticos e processos de recuperação melhorada de petróleo. Estes polímeros são constituídos de uma cadeia principal hidrofílica e alguns substituintes hidrofóbicos, que podem se encontrar distribuídos de forma aleatória ao longo da cadeia polimérica ou nas suas extremidades ${ }^{[2]}$. Devido a sua estrutura anfifílica, esses polímeros podem exibir atividade superficial e adsorver em interfaces ${ }^{[3-9]}$. Esta atividade interfacial pode ser de grande interesse tecnológico nas mais variadas aplicações, tais como obtenção de emulsões utilizadas em formulações cosméticas ${ }^{[10]}$, modificação da superficie de partículas de negro de fumo ${ }^{[11]}$ ou diminuição seletiva da permeabilidade de reservatórios de petróleo ${ }^{[12]}$.
Dentre as aplicações citadas, a de interesse desse trabalho se constitui na utilização desses polímeros solúveis em água em algumas operações de produção de petróleo, com o intuito de diminuir a permeabilidade relativa da rocha-reservatório à água sem interferir na permeabilidade relativa ao óleo, diminuindo, dessa forma, a produção de água nos poços produtores de petróleo ${ }^{[13]}$. Esse efeito é alcançado devido à capacidade de adsorção desses polímeros no interior dos poros das rochas-reservatórios ${ }^{[14]}$. Para esse tipo de aplicação, a adsorção polimérica pode ser medida em condições dinâmicas ou estáticas. A adsorção dinâmica é avaliada a partir da injeção da solução polimérica em uma amostra de rocha consolidada, seguida de medidas de concentração do polímero na solução efluente ${ }^{[15]}$. Por sua vez, a medida da adsorção estática é realizada através do contato da solução polimérica com uma amostra de rocha não-consolidada, até que a concentração do sobrenadante se torne constante ${ }^{[9,16]}$.

Diversos fatores influenciam na quantidade de polímero adsorvida, sendo os principais o tipo de polímero e suas pro-

Autor para correspondência: Rosangela B. Garcia, Departamento de Química, UFRN, Caixa Postal 1662, CEP: 59078-970, Natal, RN, Brasil. E-mail: balaban@digi.com.br 
priedades, o tipo de solvente e o tipo de substrato ${ }^{[15]}$. O tempo necessário para se atingir o equilíbrio de adsorção varia de um polímero para outro, dependendo das características da cadeia polimérica, e, por isso, não há uma metodologia padrão para a realização desse teste, sendo necessário adaptar as condições de análise ao polímero estudado.

Nesse artigo são apresentados os resultados dos testes de adsorção estática, realizados a partir de uma poliacrilamida hidrofobicamente modificada pela inserção do monômero N,N-dihexilacrilamida, visando a determinação das condições necessárias para que se atinja o equilíbrio real da quantidade adsorvida para cada concentração polimérica.

\section{Experimental}

\section{Materiais}

Foi utilizada uma amostra de poliacrilamida sintetizada em nosso laboratório a partir da copolimerização micelar de acrilamida e N,N-dihexilacrilamida, denominada PADHA, cuja síntese e caracterização encontram-se descritos em trabalhos anteriores ${ }^{[17,18]}$.

O polímero estudado (PADHA) foi previamente purificado e caracterizado por viscosimetria e ressonância magnética nuclear de hidrogênio - $\mathrm{RMN}{ }^{1} \mathrm{H}^{[18]}$. A quantidade relativa de monômero hidrofóbico $\mathrm{N}, \mathrm{N}$-dihexilacrilamida foi de $4,0 \mathrm{~mol} \%$ e a viscosidade intrínseca de $414 \mathrm{~mL} \cdot \mathrm{g}^{-1}$, a $25 \pm 0,01{ }^{\circ} \mathrm{C}$, em água destilada.

Utilizou-se como substrato um arenito da formação Botucatu (Ribeirão Preto, SP), previamente desagregado e calcinado, com diâmetro de partícula entre 150 e $250 \mu \mathrm{m}$.

\section{Testes de adsorção em arenito não-consolidado}

\section{Preparação das soluções}

As soluções do copolímero PADHA foram obtidas pela diluição do meio reacional com soluções aquosas de $\mathrm{NaCl}$, de forma que a concentração final deste sal fosse de 300 g.L $\mathrm{L}^{-1}$, e a concentração de polímero variasse entre 0,1 e $1 \mathrm{~g} . \mathrm{L}^{-1}$. A adsorção do polímero foi avaliada na presença de sal (300 g. $\mathrm{L}^{-1} \mathrm{NaCl}$ ) para simular o meio encontrado nos reservatórios de petróleo de alta salinidade ${ }^{[13]}$.

\section{rocha}

Determinação do grau de adsorção do polímero na

A determinação do teor de polímero adsorvido na superfície do arenito foi realizada através da diferença entre a concentração inicial e final da solução de polímero colocada em contato com o arenito. As concentrações do polímero em solução foram obtidas a partir de um método reológico ${ }^{[12,19-21]}$, que consistiu em determinar a concentração através de curvas de calibração, geradas a partir da tensão de cisalhamento medida para diferentes soluções de concentração conhecida do polímero, a uma taxa de cisalhamento constante de $850 \mathrm{~s}^{-1}$. Foi utilizado um sensor do tipo cilindros coaxiais DG41, em reômetro RheoStress da Haake, modelo RS 150, acoplado a um banho termostatizado à temperatura de $25^{\circ} \mathrm{C}$. Os experimentos foram realizados em duplicata e a média dos resultados foi utilizada na preparação da curva de calibração. $\mathrm{O}$ coeficiente de variação oscilou em torno de $10 \%$.

\section{Determinação das condições ideais para a construção da isoterma de adsorção}

Para se determinar a cinética de adsorção e a razão sólido/ líquido (s/l) adequadas para a construção da isoterma de adsorção, soluções poliméricas com concentração de 0,4 g.L $\mathrm{L}^{-1}$ (concentração frequentemente usada em poços injetores nos campos de petróleo) foram colocadas em contato com amostras de arenito e mantidas sob agitação, de acordo com as condições descritas abaixo.

A cinética de adsorção foi obtida colocando-se $25 \mathrm{~mL}$ de solução polimérica, à concentração de 0,4 g.L.-1, em contato com $5 \mathrm{~g}$ de várias amostras de arenito, o que corresponde a uma razão sólido/líquido (s/l) de $2 \times 10^{-1}$. A quantidade de polímero adsorvido foi determinada em diferentes tempos: $4,8,16,30$ e 48 horas.

$\mathrm{O}$ experimento foi repetido utilizando-se diferentes razões s/1 $\left(2 \times 10^{-2} ; 1 \times 10^{-2} ; 7 \times 10^{-3} ; 4 \times 10^{-3} ; 1 \times 10^{-3} \mathrm{e}\right.$ $1 \times 10^{-4} \mathrm{~g} \cdot \mathrm{mL}^{-1}$ ) para a determinação da razão s/l adequada para a PADHA. Nessa etapa, as suspensões foram agitadas por 24 horas.

Ao final do tempo de contato entre a solução polimérica e o mineral, as suspensões foram centrifugadas $(15.000 \mathrm{rpm}$, 10 minutos, $25^{\circ} \mathrm{C}$ ) em uma centrífuga refrigerada Himac CR $21 \mathrm{G}$, para promover a separação entre o sólido e o líquido. O sobrenadante foi filtrado em membranas Millipore de acetato de celulose com diâmetro de poro $3,0,8$ e 0,45 $\mu \mathrm{m}$ e a concentração de equilíbrio do polímero no sobrenadante foi determinada pelo método reológico, como descrito anteriormente. A quantidade de polímero adsorvida foi calculada a partir da diferença entre a concentração inicial e a concentração final do polímero no sobrenadante, após o contato com o mineral.

\section{Determinação da isoterma}

A isoterma de adsorção foi obtida a partir de soluções do polímero a concentrações de 0,2, 0,6 e 1 g.L L $^{-1}$. Essa faixa de concentração foi selecionada por ser frequentemente usada nas operações de controle de produção de água nos poços produtores de petróleo. As soluções foram colocadas em contato com amostras de arenito, na razão s/l de $1,2 \times 10^{-2}$, e agitadas por 24 horas. As concentrações finais foram determinadas pelo método reológico, usando a curva de calibração descrita anteriormente, após a centrifugação das suspensões polímero/arenito e filtração do sobrenadante. A quantidade de polímero adsorvido foi calculada pela diferença entre a concentração inicial do polímero e a sua concentração no sobrenadante, após o contato com a rocha. 


\section{Resultados e Discussão}

\section{Curva de calibração para determinação da concentração de polímero em solução}

Foi utilizado um método indireto a partir de medidas reológicas. Basicamente, usou-se uma curva de calibração de concentração vs. tensão de cisalhamento, conforme mostrado na Figura 1. Na taxa de cisalhamento constante utilizada $\left(850 \mathrm{~s}^{-1}\right)$, as soluções poliméricas estudadas apresentaram comportamento newtoniano. Sendo assim, a tensão de cisalhamento obtida para cada solução pôde ser considerada dependente apenas da concentração do polímero. A curva de calibração foi gerada a partir de uma regressão polinominal de segundo grau e apresentou coeficiente de correlação próximo de um. Isso significa que houve um bom ajuste da curva aos pontos experimentais. A partir da curva de calibração, foi obtida a relação entre concentração do polímero (c) e tensão de cisalhamento $(\tau)$ (Equação 1). Essa equação foi usada para a determinação da concentração do polímero nos sobrenadantes.

$$
c=-0,4993 \tau^{2}+2,8705 \tau-3,1245
$$

\section{Cinética de adsorção}

A Figura 2 mostra que a adsorção da PADHA em arenito tem uma velocidade intermediária, quando comparada com os valores citados na literatura, que necessitam de alguns minutos a dias para o sistema atingir o equilíbrio ${ }^{[14]}$. Observou-se que o copolímero PADHA necessitou de cerca de 16 horas para

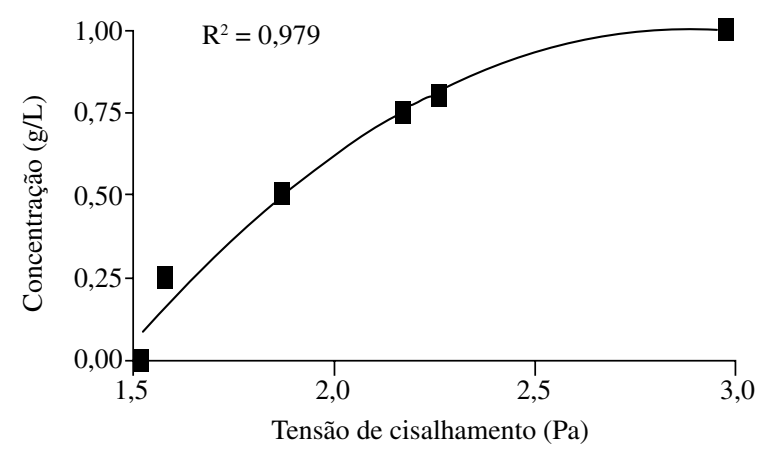

Figura 1. Curva de calibração de adsorção estática em arenito não-consolidado para PADHA.

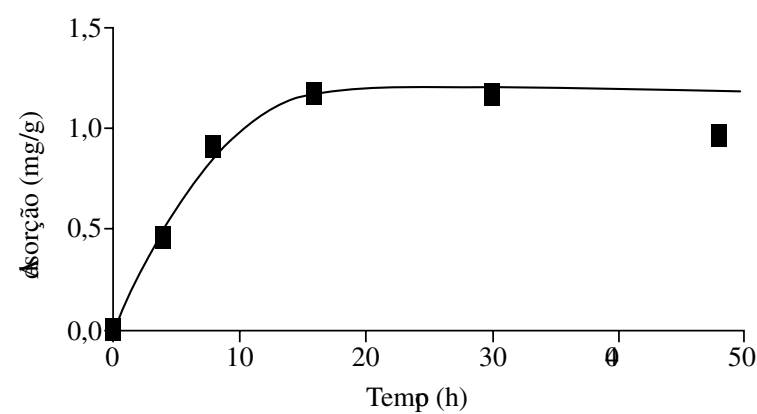

Figura 2. Variação da adsorção com o tempo de contato para uma solução de PADHA a 0,40 g.L $\mathrm{L}^{-1}$, em uma razão sólido/líquido de 0,2 g. $\mathrm{mL}^{-1}$. apresentar um patamar. Sendo assim, as análises seguintes foram feitas com um tempo de contato entre o arenito e a solução polimérica de 24 horas, de forma a se ter certeza que o sistema teve tempo suficiente para atingir o equilíbrio. Não se praticou tempos maiores de contato entre a solução polimérica e o arenito para evitar eventual degradação por cisalhamento, dada a agitação intensa à que o meio contendo o polímero e o arenito é submetido, necessária para manter o mineral em suspensão.

\section{Razão sólido/líquido (s/l)}

Durante a determinação da razão s/l ideal, observou-se que os valores de adsorção variavam de acordo com a relação entre a massa de substrato e o volume de solução polimérica, como pode ser visto na Figura 3. E estes valores diminuíram à medida que a razão $\mathrm{s} / \mathrm{l}$ aumentou até $4 \times 10^{-3}$. Esse resultado ocorreu, provavelmente, devido à agregação das partículas do mineral, causada pela adsorção do polímero de alta massa molar, levando a uma progressiva diminuição da superfície acessível ao polímero para a adsorção ${ }^{[5]}$. A partir da razão s/1 $4 \times 10^{-3}$, o valor da adsorção passou a ficar em torno de $17 \mathrm{mg} / \mathrm{g}$ (média dos 4 últimos pontos da curva na Figura 3). De acordo com esses resultados, construiu-se a isoterma de adsorção utilizando-se a razão s/l de 1,2 x 10-2 , para evitar que a razão entre o volume de solução polimérica e a quantidade de arenito levasse a variações nos valores medidos para a construção da isoterma de adsorção.

\section{Isoterma de adsorção}

A Figura 4 mostra a isoterma de adsorção obtida a partir do método reológico, para a amostra PADHA.

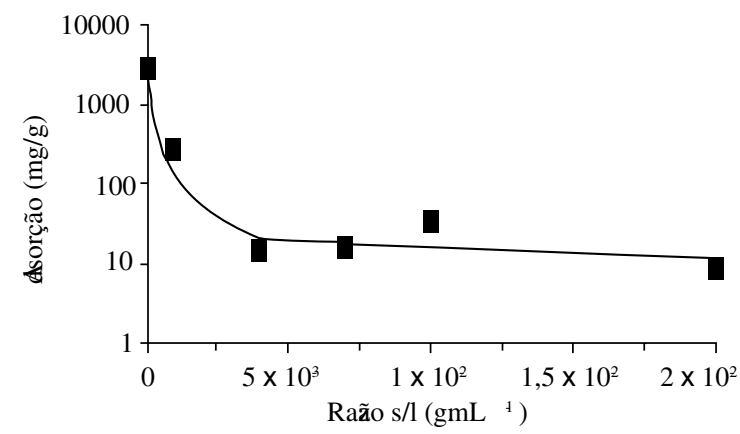

Figura 3. Variação da adsorção com a razão sólido/líquido para a PADHA, à concentração de polímero de 0,40 g.L $\mathrm{L}^{-1}$.

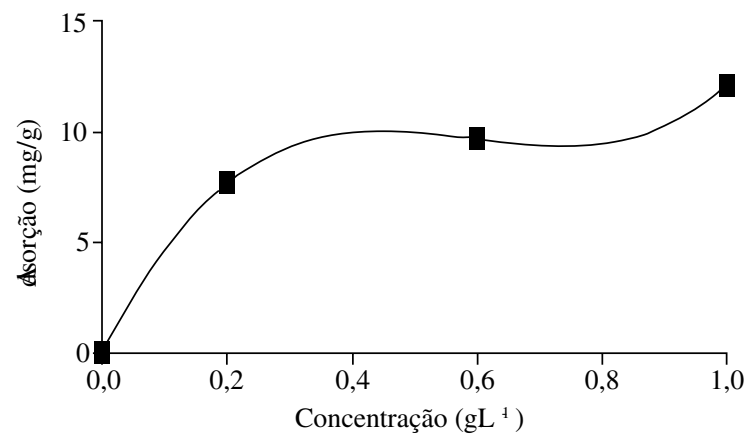

Figura 4. Isoterma de adsorção da PADHA sobre arenito, em 300 g.L. $\mathrm{L}^{-1}$ de $\mathrm{NaCl}$ e razão s/1 1,2 $\times 10^{-2} \mathrm{~g} \cdot \mathrm{mL}^{-1}$. 
Não se observou a formação de um patamar para a PADHA, que seria o comportamento clássico esperado para homopoliacrilamidas $^{[5,15]}$, e a quantidade adsorvida aumentou continuamente com a concentração do polímero em solução. Argillier e colaboradores ${ }^{[5]}$ já haviam observado que poliacrilamidas hidrofobicamente modificadas apresentam um perfil de adsorção diferente das poliacrilamidas tradicionais, provavelmente, porque sua adsorção ocorra em diversas camadas sobrepostas. A primeira camada adsorvida seria composta por segmentos poliméricos diretamente em contato com a superfície mineral, e as camadas superiores seriam formadas por segmentos ligados aos segmentos adsorvidos através de interações hidrofóbicas. Essa superposição de camadas explicaria o porquê do copolímero não atingir um patamar com o aumento da sua concentração, assim como seus níveis de adsorção serem bem mais elevados que os citados na literatura para as poliacrilamidas ${ }^{[15]}$. Contudo, valores de adsorção acima de $1 \mathrm{mg} / \mathrm{g}$ são esperados para polímeros hidrofobicamente modificados ${ }^{[5]}$.

$\mathrm{O}$ uso de uma solução extremamente salina $(\mathrm{NaCl}$ 300 g. $\left.\mathrm{L}^{-1}\right)$ também pode ter influenciado os valores de adsorção medidos nesse trabalho ${ }^{[22]}$, pois a presença de sais leva a uma diminuição na qualidade do solvente para polímeros hidrofobicamente modificados, já que há uma tendência de parte das moléculas de água solvatarem preferencialmente os íons ${ }^{[6]}$, o que torna a interação entre o polímero e a superfície sólida ainda mais significativa.

\section{Conclusões}

Os testes de adsorção estática sobre arenito não-consolidado mostraram que a PADHA adsorve mais que as poliacrilamidas não-modificadas descritas na literatura. Provavelmente, isso ocorre porque sua adsorção se dá em múltiplas camadas, graças à interação entre meros hidrofóbicos das moléculas livres e das moléculas adsorvidas sobre a superfície da rocha. Devido à maior adsorção de suas moléculas sobre rochas do tipo arenito, o copolímero analisado demonstrou ter potencial para utilização em processos de recuperação melhorada de petróleo, em poços de alta salinidade e alta permeabilidade, como redutores da permeabilidade da rochareservatório à água e para a diminuição da produção de água em poços produtores de petróleo, como será mostrado em uma próxima publicação ${ }^{[23]}$.

\section{Agradecimentos}

Os autores agradecem à FINEP-CTPETRO, PETROBRAS e PRH-ANP/MCT pelo auxílio financeiro.

\section{Referências Bibliográficas}

1. Peit, F.; Iliopoulos, I.; Audebert, R. \& Szonyl, S. - Langmuir, 13, p.4229 (1997).

2. Petit-Agnely, F.; Iliopoulos, I. \& Zana, R. - Langmuir, 16, p.9921 (2000).
3. Yahya, G. O. \& Hamad, E. Z. - lymer, 36, p.3705 (1995).

4. Yahya, G. O.; Asrof, S. K. \& Hamad, E. Z. - Polymer, 37, p.1183 (1996).

5. Argillier, J. F.; Audibert, A.; Lecourtier, J.; Moan, M. \& Rousseau, L. - Colloids Surf., A, 113, p.247 (1996).

6. Yahaya, G. O.; Ahdab, A. A.; Ali, S. A.; Abu-Sharkh, B. F. \& Hamad, E. Z. - Polymer, 42, p.3363 (2001).

7. Fischer, A.; Brembilla, A. \& Lochon, P. - Polymer, 42, p.1441 (2001).

8. Córdova, M.; Mogollón, J. L.; Molero, H. \& Navas, M. - Society of Petroleum Engineers Journal, SPE 75210 (2002).

9. Chauveteau, G \& Lecourtier, J. - "Propagation of Polymer Slugs through Adsorbent Porous Media", in: Water-Soluble Polymers for Petroleum Recovery, Stahl, G \& Schulz, D. N. (ed.), Plenum Press, New York (1998).

10. Akiyama, E., Kashimoto, A., Hotta, H. \& Kitsuki, T. J. Colloid Interface Sci., 300, p.141 (2006).

11. Ridaoui, H., Jada, A., Vidal, L. \& Donnet, J.-B. - Colloids Surf., A, 278, p.149 (2006).

12. Taylor, K. C. \& Nasr-E1-Din, H. A. - J. Pet. Sci. Eng, 12, p.9 (1994).

13. Ranjbar, M. \& Schaffie, M., - J. Pet. Sci. Eng, 26, p.133 (2000).

14. Grattoni, C. A.; Luckham, P. F., Jing, X. D.; Norman, L. \& Zimmerman, R. W. - J. Pet. Sci. Eng, 45, p.233 (2004).

15. Sorbie, K. S. - "Polymer-Improved Oil Recovery", Blackie, London (1991).

16. Mezzomo, R. F.; Moczydlower, P.; Sanmartin, A. N. \& Araujo, C. H. V. - Society of Petroleum Engineers Journal, SPE 75204 (2002).

17. Camail, M.; Margaillan, A.; Martin, I.; Papailhou, A. L. \& Vernet, J. L. - Eur. Polym. J., 36, p.1853 (2000).

18. Maia, A. M. S.; Costa, M.; Borsali, R. \& Garcia, R. B. - Macromol. Symp., 229, p.217 (2005).

19. Maia, A. M. S., Galvão, L. P. F. C.; Vidal, R. R. L. \& Garcia, R. B. - "Testes de Adsorção de Poliacrilamida em Arenito", in: Anais do XV Congresso Brasileiro de Engenharia e Ciência dos Materiais, p.4644, Natal - RN, nov (2002).

20. Maia, A. M. S., Galvão, L. P. F. C.; Vidal, R. R. L. \& Garcia, R. B. - "Estudo da Adsorção de Poliacrilamidas Aniônicas em Arenito Não-consolidado", in: Anais do $2^{\circ}$ Congresso Brasileiro de P\& D em Petróleo e Gás, Rio de Janeiro - RJ, jun (2003).

21. Maia, A. M. S.; Vidal, R. R. L. \& Garcia, R. B. - "Utilização de reologia na obtenção de isotermas de adsorção de polímeros sobre arenitos", in: Anais do $5^{\circ}$ Encontro Brasileiro sobre Adsorção, p.164, Natal - RN, jul (2004).

22. Smith, F. W. - J. Pet. Tech. 22, p.48 (1970).

23. Maia, A. M. S., Chagas, E. F., Costa, M. \& Garcia, R. B. - Evaluation of the acrylamide- $N, N$-dihexylacrylamide copolymer performance for enhanced oil recovery (manuscrito em fase de preparação).

Enviado: 03/02/06

Reenviado: 09/10/06

Aprovado: 20/11/06 